\title{
On the accuracy limits of plate theories for vibro-acoustic predictions
}

\author{
U. Arasan ${ }^{\mathrm{a}, \mathrm{c}, \mathrm{d}, *}$, Fabien Marchetti ${ }^{\mathrm{a}}$, Fabien Chevillotte ${ }^{\mathrm{a}, *}$, Gregor Tanner $^{\mathrm{b}}$, \\ Dimitrios Chronopoulos ${ }^{\mathrm{c}}$, Emmanuel Gourdon ${ }^{\mathrm{d}}$ \\ ${ }^{a}$ Matelys - Research Lab, F-69120 Vaulx-en-Velin, France \\ ${ }^{b}$ School of Mathematical Sciences, University of Nottingham, University Park, NG7 \\ $2 R D, U K$ \\ ${ }^{c}$ Institute for Aerospace Technology and The Composites Group, University of \\ Nottingham, University Park, NG7 2RD, UK \\ ${ }^{d}$ Université de Lyon, ENTPE, LTDS UMR CNRS 5513, 3 rue Maurice Audin, 69518 \\ Vaulx-en-Velin Cedex, France
}

\begin{abstract}
Several vibro-acoustic models for either single wall or multi-layer constructions are based on classical plate and first order shear deformation theories. The equivalent or condensed plate models employ the thin plate model to extract the dynamic mechanical properties of the multi-layer system considering only flexural and shear motions for the structure under investigation. Since these plate models do not account for the compressional or symmetric motion of the structure, both thin and thick plate theories encounter limitations for mid to high frequency predictions depending on the structures considered. In this work, analytical expressions for the frequency limit of thin and thick plate theories are derived for an elastic layer of isotropic material from the analyses of wavenumbers and admittances. Additionally, refined expressions for coincidence and critical frequencies are presented. Validation of these frequency limits are made by comparing the transmission loss (TL) obtained from both plate theories with the TL computed through the theory of elasticity for a range of thin/thick and soft/stiff materials.
\end{abstract}

Keywords: Wavenumbers, Thin plate theory, Thick plate theory, Elasticity theory, Coincidence frequency, Critical frequency

\footnotetext{
*Corresponding authors:

Email addresses: arasan.uthaysuriyan@matelys.com (U. Arasan), fabien.chevillotte@matelys.com (Fabien Chevillotte)
} 


\section{NOMENCLATURE}

\begin{tabular}{|c|c|c|c|}
\hline$h$ & Thickness & $\theta$ & Incident angle \\
\hline$k_{0}$ & Wavenumber in the air & $k_{t}$ & Transverse wavenumber \\
\hline$k_{p}$ & Natural propagating wavenumber & $k_{b}$ & Bending wavenumber \\
\hline$k_{s}$ & Corrected shear wavenumber & $k_{m}$ & Membrane wavenumber \\
\hline$\delta_{l}$ & Longitudinal wavenumber & $\delta_{s}$ & Shear wavenumber \\
\hline$\omega$ & Circular frequency & $f$ & Frequency \\
\hline$f_{\text {cut-on }}$ & Cut-on frequency & $f_{\text {comp }}$ & Compressional frequency \\
\hline$f_{\text {thin/thick }}$ & $\begin{array}{l}\text { Frequency limit of thin plate the- } \\
\text { ory }\end{array}$ & $f_{\text {coinc }_{\text {thin }}}$ & $\begin{array}{l}\text { Coincidence frequency of a thin } \\
\text { plate }\end{array}$ \\
\hline$f_{\text {coinc }_{\text {thick }}}$ & $\begin{array}{l}\text { Coincidence frequency of a thick } \\
\text { plate }\end{array}$ & $f_{\text {cri }_{\text {thin }}}$ & Critical frequency of a thin plate \\
\hline$f_{\text {cri }_{\text {thick }}}$ & Critical frequency of a thick plate & $f_{\text {plate/solid }_{\mathrm{oi}}}$ & $\begin{array}{l}\text { Frequency limit of plate theories } \\
\text { for oblique incidence }\end{array}$ \\
\hline$f_{\text {plate } / \text { solid }_{\text {df }}}$ & $\begin{array}{l}\text { Frequency limit of plate theories } \\
\text { for diffuse field }\end{array}$ & $c_{0}$ & Speed of sound in the air \\
\hline$V^{P}$ & State vector of a plate & $V^{E S}$ & State vector of an elastic layer \\
\hline$p$ & Pressure at a point & $u$ & Transverse velocity at a point \\
\hline$v$ & Normal velocity at a point & $\sigma_{z z}$ & Normal stress at a point \\
\hline$\sigma_{x z}$ & Shear stress at a point & {$\left[T^{P}\right]$} & Transfer matrix of a plate \\
\hline$\left[T^{E S}\right]$ & Transfer matrix of an elastic layer & $m_{s}$ & Mass density per unit area \\
\hline$\rho$ & Volume density of elastic layer & $\rho_{0}$ & Volume density of air \\
\hline$D$ & Bending stiffness & $E$ & Young's modulus \\
\hline$K$ & Compressional modulus & $\eta$ & Loss factor \\
\hline$\nu$ & Poisson's ratio & $G$ & Shear modulus \\
\hline$G^{*}$ & Corrected shear modulus & $\kappa$ & Shear correction factor \\
\hline$I_{z}$ & Mass moment of inertia & $\lambda, \mu$ & Lamé coefficients \\
\hline$\tau$ & Transmission factor & $Z_{P}$ & $\begin{array}{l}\text { Anti-symmetric impedance of a } \\
\text { plate }\end{array}$ \\
\hline$Z_{s}$ & Symmetric impedance & $Z_{a}$ & Anti-symmetric impedance \\
\hline$Y_{s}$ & Symmetric admittance & $Y_{a}$ & Anti-symmetric admittance \\
\hline$\tilde{Y}_{s}$ & $\begin{array}{l}\text { Approximated symmetric admit- } \\
\text { tance }\end{array}$ & $Z_{0}$ & Characteristic impedance of air \\
\hline$C_{k}$ & $\begin{array}{l}\text { Ratio between bending and shear } \\
\text { wavenumbers }\end{array}$ & $C_{y}$ & $\begin{array}{l}\text { Minimum value of ratio between } \\
\text { anti-symmetric and symmetric } \\
\text { admittances }\end{array}$ \\
\hline$\epsilon$ & Error percentage & $\lambda_{l}$ & Longitudinal wavelength \\
\hline
\end{tabular}

\section{Introduction}

When studying the sound insulation of a wall, the main acoustic indicator is the transmission loss (TL) which is controlled by the combination of several fundamental vibrating modes of the wall. For example, a typical sound transmission problem encountered in building applications is presented schematically in Fig. 1 along with its vibrating modes as the acoustic energy transmitted through the wall depends on its vibro-acoustic behaviour. Although the wall vibrates in a complex manner for the given acoustic excitation, this complex motion can be obtained by superposing the fundamental 


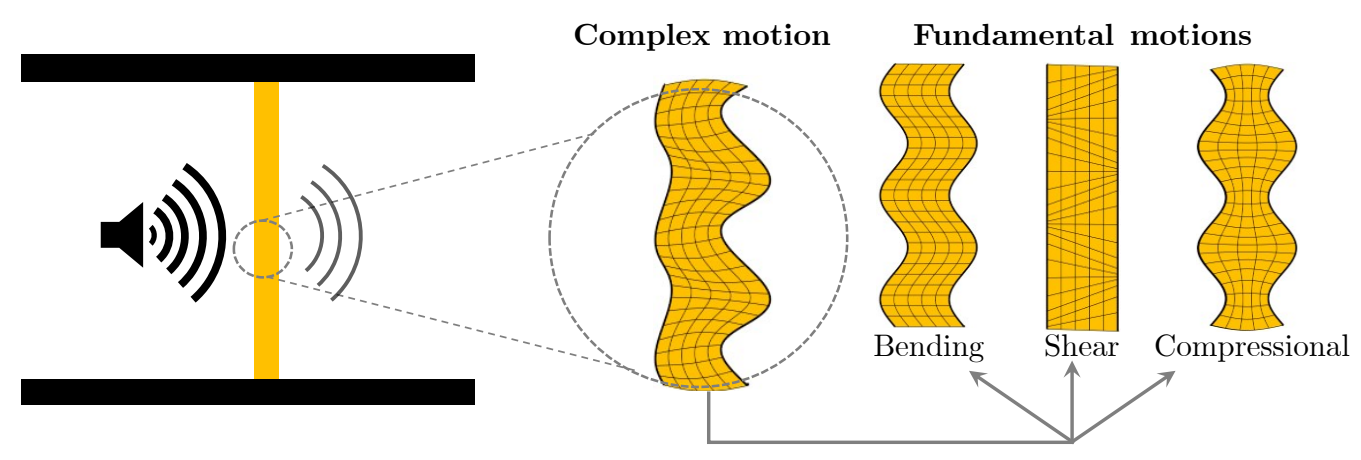

Figure 1: Schematic representation of vibrating motion of a single wall subjected to acoustic excitation.

motions (bending, shear and compressional/dilatational motions). Generally, looking at the TL characteristics of a single wall as a function of frequency, three regions can be identified which are controlled by the mass, damping and stiffness of the wall respectively. The mass and stiffness control zones are separated by a critical region where strong reduction of transmission loss is observed. This critical zone is characterized by its frequency which is called the critical frequency. Various vibro-acoustic models of varying complexity have been developed to predict the sound insulation properties of plate structures, especially with regard to noise attenuation problems. An early model was developed by Cremer [1] which was applied to the computation of the TL across infinite, thin walls. Related work approaching the same problem of computing the acoustic insulation indicators of a thin wall are presented in [2-6]. In Cremer's model, it is assumed that the motion of the plate is described only by the bending wave equation, which is based on the classical plate theory [7].

Davy [8] argues that Cremer's model can only be used below the critical frequency since most of the approximations are not valid within and above the critical region. Improving on Cremer's theory, Heckl and Donner [9] developed a model based on the first order shear deformation theory (FSDT) [10-12] which could be applied to thicker walls to compute sound TL. In this model, motion due the transverse shear is also included along with the flexural motion of the plate. The corrected TL expression accounting for shear deformation of the plate can be found in [13]. Heckl and Donner [9] point out that their model is valid only at frequencies well below the first dilatational or compressional frequency of the plate. This is due to the fact that the FSDT does not account for the thickness stretch motion of the plate as it assumes constant velocity at all points through the thickness direction. Consequently, the symmetric motion of the plate is not taken into account 
in zero, first and higher order plate theories. This may lead to deviations between the predictions and the actual motion of the plate at higher frequencies, especially when the material is soft. In the work published by Ljunggren $[13,14]$, the general expression to compute the TL of an infinite wall with arbitrary uniform thickness is given, accounting for both antisymmetric and symmetric motions of the plate.

In recent years, instead of single wall structures, multi-layered structures have been used widely for better sound comfort and noise attenuation. These structures provide the designers with more choices for tuning the vibro-acoustic performance leading to better sound insulation characteristics. Advanced composite structures are one example of multi-layer systems that are progressively used in different fields such as the space, energy and aeronautical industries. In transport and construction industries, sandwich structures are widely used as they provide high stiffness with significantly low weight. In most cases, two face sheets are bonded with a viscoelastic layer to improve the overall damping response of the structure. There exist a large number of theoretical models dedicated to the analysis of the behaviour of multi-layer structures. According to Carrera [15, 16], these models can be classified into three major categories as: 1) Equivalent Single Layer (ESL) models, 2) Layer Wise (LW) models and 3) Hybrid or Zig-Zag models. ESL models describe the dynamics of the multi-layer plate in terms of the displacement field of an equivalent layer. It is noted that, due to this kind of displacement description, the number of layers present in the system do not influence the displacement functions which gives great flexibility in using shear deformation theories of order one [10-12] and higher [17-20]. Layer Wise (LW) models describe the displacement field in each layer [21-28]; as a consequence, this type of model requires higher computational effort as the number of unknowns increases with the number of layers present in the structure. Hybrid or Zig-Zag models make use of advantages from the previous two categories. Although the displacement field is defined in each layer (similar to the LW models), the interface continuity conditions between two adjacent layers results in a lower number of unknown functions (as in ESL models) which do not depend on the number of layers present in the structure. The reader may refer to [29-34] which use this family of models to describe the dynamic response of multi-layer systems.

Since industrial multi-layer structures are manufactured with a diversity of materials, they naturally increase the computational burden for detailed finite element modelling and it is therefore of interest to condense the behaviour of the multi-layer system into a single layer. A simplified equivalent thin plate model was developed in [35-37] for sandwich structures with a viscoelastic core. Guyader and Cacciolati [38] have developed an equivalent 
thin plate model for the multi-layer structure of isotropic layers. Following a similar path, Marchetti et al. [39] have recently developed an equivalent thin plate model for laminated structures of orthotropic layers. The aim of the equivalent plate models is to find the frequency dependent mechanical parameters of the equivalent thin plate that incorporates the bending and shear motions of the multi-layer structures. Since plate theories do not account for the dilatational or compressional motion of the structure, finding a frequency domain of validity is necessary to safely use these equivalent plate models.

The reader may note that the words 'frequency limit' of a theory refer here to the frequency up to which the theory can be applied within pre-defined accuracy intervals for computing the desired acoustic indicators. Although in structural mechanics and dynamics, thin and thick plates are distinguished based on the thickness to lateral dimensions ratio [40, 41], such rules may not be sufficient for vibro-acoustic calculations as they depend on the material properties of the plate as well. Additionally, although plate theories (both for thin and thick plates) are commonly employed in computing the acoustic indicators of infinite and finite walls, there is currently no clear-cut frequency limit to restrict the applicability of these theories. Qualitative and approximate frequency limits are given in the literature but it is often a tedious task to find an analytical expression for applicability limits.

In this work, we derive for the first time analytical expressions for the applicability limits in the spectral domain for thin and thick plate theories. Through analysis of the propagating wavenumbers and admittances of the investigated panels, we quantify the expected accuracy of each theory. The paper is organized as follows: Sec. 2 describes the theories behind propagating wavenumbers inside thin, thick plates and elastic solids. The theory of elastic solids is treated as a reference since it describes the complete motion of an infinite layer [42]. In Sec. 3, expressions for the limits of applicability of thin plate theories are discussed by comparing propagating wavenumbers of thin and thick plate theories. Additionally, refined expressions for the coincidence and critical frequencies are also presented. In Sec. 4, an expression for the frequency limit of applicability of plate theories is derived in all generality by comparing the order of magnitudes of both symmetric and anti-symmetric admittances of the plate. In Sec. 5, analytical expressions for the frequency limits of different plate theories are presented along with sound transmission loss computations for classical industrial materials for validation purposes. 


\section{Vibro-acoustic models for an elastic isotropic layer}

We start by giving the theoretical background for some commonly used theories such as the Love-Kirchoff [7, 43] or the Reissner-Mindlin [10-12] theory used to describe the vibro-acoustic behaviour of isotropic, single wall structures. In subsequent sections, these theories are compared and their limitations are discussed.

\subsection{Wave propagation in an elastic isotropic plate}

Let us consider an infinitely extended elastic medium with thickness $h$ as shown in Fig. 2. An oblique wave is assumed to be impinging upon one of the surfaces of the elastic medium with an incident angle $\theta$. For the sake of simplicity, the incident plane wave is assumed to be in the $x-z$ plane. Depending on the material properties of the elastic layer, various types of

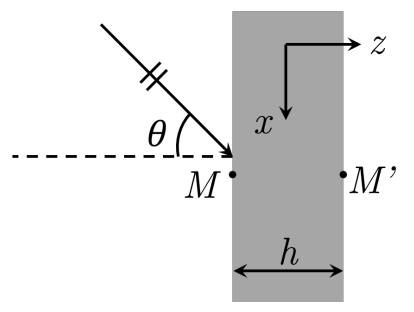

Figure 2: An oblique plane wave impinging on an infinitely extending elastic isotropic layer with incident angle $\theta$.

wave propagation are possible. The wave propagation in the $x-z$ plane will have wave vector components along the $x$ and $z$ axes for each wave. The incident wave in free air is exciting waves inside the elastic medium; continuity across the interface demands that the transverse or $x$ component of the wave vector, $k_{t}$, for waves propagating in the plate and in air are equal. We have

$$
k_{t}=k_{0} \sin \theta=\frac{\omega}{c_{0}} \sin \theta,
$$

where $k_{0}$ is the wavenumber in free air, $\omega=2 \pi f$ is the circular frequency of the incident wave and $c_{0}$ is the speed of sound in air. If the acoustic field in the elastic layer (Fig. 2) is described by the state vector $V^{P}=\{p, v\}^{T}$ (where the superscript $P$ denotes plate, $p$ is the acoustic pressure and $v$, the particle velocity normal to the interface), then the general form of the transfer matrix for plate theories discussed can be expressed by the following equation:

$$
V_{M}^{P}=\left[T^{P}\right] V_{M^{\prime}}^{P}=\left[\begin{array}{cc}
1 & Z_{P} \\
0 & 1
\end{array}\right] V_{M^{\prime}}^{P}
$$


Here, $M$ and $M^{\prime}$ are points on the left and right hand side on the surface of the elastic medium, respectively and $Z_{P}$ is the anti-symmetric mechanical impedance of the plate, that is, the ratio of the differential complex sound pressure across the plate to the complex plate velocity; it is expressed as below based on the theory adopted, that is,

$$
Z_{P}=\left\{\begin{array}{l}
Z_{\text {thin }}=j \omega m_{s}\left(1-\frac{D k_{t}^{4}}{\omega^{2} m_{s}}\right) \\
Z_{\text {thick }}=\frac{k_{t}^{4} D-m_{s} \omega^{2}+\left(\frac{I_{z} m_{s}}{G^{*} h} \omega^{2}-k_{t}^{2} \frac{D m_{s}}{G^{*} h}\right) \omega^{2}-k_{t}^{2} I_{z} \omega^{2}}{j \omega\left(\frac{k_{t}^{2} D-I_{z} \omega^{2}}{G^{*} h}+1\right)},
\end{array}\right.
$$

where Love-Kirchoff theory applies for thin plates and Reissner-Mindlin theory for thick plates. Here, $m_{s}$ is the mass density per unit area,

$$
D=\frac{E(1+\mathrm{j} \eta) h^{3}}{12\left(1-\nu^{2}\right)}
$$

is the bending stiffness, $E$ is the Young's modulus, $\mathrm{j}=\sqrt{-1}, \eta$ is the loss/damping factor, $\nu$ is the Poisson's ratio, $G^{*}=\kappa G$ with $G$, the shear modulus of the plate and $\kappa$, the shear correction factor accounting for the transverse shear distribution. Furthermore,

$$
I_{z}=\frac{\rho h^{3}}{12}
$$

is the mass moment of inertia of the plate and $\rho=m_{s} / h$ is the volume density of the plate material. Detailed derivations of the mechanical impedances given in Eq. (3) can, for example, be found in the book by Cremer and Heckl [44].

When using the thick plate theory, the shear correction factor, $\kappa$, is substituted with different values/expressions by different authors. For example, Reissner [11] and Mindlin [10] used the values $5 / 6$ and $\pi^{2} / 12$, respectively, for the shear correction factor, whereas Heckl and Donner [9] used the following expression given by Magrab [45],

$$
\kappa=\left(\frac{0.87+1.12 \nu}{1+\nu}\right)^{2}
$$

which is a function of the Poisson's ratio of the plate. In this article, the value of $\kappa$ is taken as $5 / 6$. 


\subsubsection{Dispersion relations}

Given the mechanical impedances of the structure, dispersion relations are often obtained by setting the impedance to zero. In other words, dispersion relations are used to understand the wave propagation in the structure under natural or free vibration conditions.

For Love-Kirchoff plates (or thin plates), by setting the mechanical impedance equal to zero, it can be observed that only one type of wave propagation is possible, that is,

$$
Z_{\text {thin }}=0 \Rightarrow k_{p}^{4} D-m_{s} \omega^{2}=0 \Rightarrow k_{p}=k_{b}=\sqrt{\omega \sqrt{\frac{m_{s}}{D}}}
$$

where $k_{b}$ corresponds to the bending wavenumber and $k_{p}$ is the natural propagating wavenumber of the plate.

For Reissner-Mindlin plates (or thick plates), the dispersion relation is obtained as

$$
Z_{\text {thick }}=0 \Rightarrow k_{p}^{4} D-m_{s} \omega^{2}+\left(\frac{I_{z} m_{s}}{G^{*} h} \omega^{2}-k_{p}^{2} \frac{D m_{s}}{G^{*} h}\right) \omega^{2}-k_{p}^{2} I_{z} \omega^{2}=0 .
$$

There are four possible solutions for the above quartic equation, that is,

$$
k_{p}= \pm \sqrt{\frac{m_{s} \omega^{2}}{2 G^{*} h}+\frac{I_{z} \omega^{2}}{2 D} \pm \sqrt{\frac{m_{s} \omega^{2}}{D}+\left(\frac{m_{s} \omega^{2}}{2 G^{*} h}-\frac{I_{z} \omega^{2}}{2 D}\right)^{2}}} .
$$

Out of the four solutions, two correspond to outgoing waves, that is, the real part of the wavenumber is positive; these are

$$
k_{p}=k_{R M_{1,2}}=\sqrt{\frac{m_{s} \omega^{2}}{2 G^{*} h}+\frac{I_{z} \omega^{2}}{2 D} \pm \sqrt{\frac{m_{s} \omega^{2}}{D}+\left(\frac{m_{s} \omega^{2}}{2 G^{*} h}-\frac{I_{z} \omega^{2}}{2 D}\right)^{2}}} .
$$

It is observed from the above equation that the propagating wavenumber $\left(k_{R M_{1}}\right)$ has different asymptotic behaviour with respect to low and high frequencies as shown in Fig. 3, here for the example of a $50 \mathrm{~mm}$ plasterboard with mechanical properties listed in Table 1. One finds in particular:

- At low frequency (or $\omega \rightarrow 0$ ), we have

$$
\frac{m_{s}}{D} \gg\left(\frac{m_{s}}{2 G^{*} h}-\frac{I_{z}}{2 D}\right)^{2} \omega^{2}
$$

which results in $k_{R M_{1}}$ tending to $k_{b}$ 


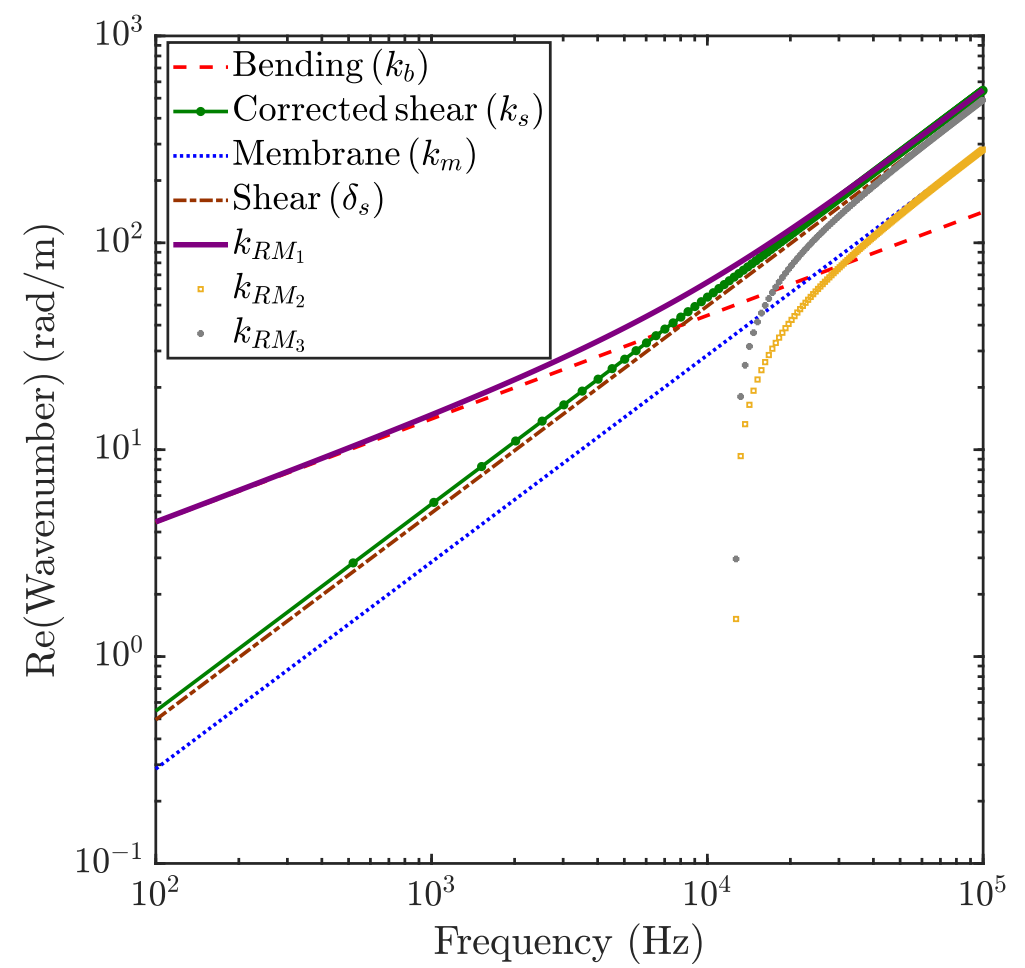

Figure 3: Propagating wavenumbers of a Reissner-Mindlin plate (50 $\mathrm{mm}$ plasterboard with mechanical properties mentioned in Table 1) and its asymptotic behaviours. It is observed that the main natural propagating wavenumber $k_{R M_{1}}$ is approaching bending $\left(k_{b}\right)$ and corrected shear wavenumbers $\left(k_{s}\right)$ at low and high frequencies respectively.

- At high frequency (or $\omega \rightarrow+\infty)$, we find

$$
\frac{m_{s}}{D} \ll\left(\frac{m_{s} \omega^{2}}{2 G^{*} h}-\frac{I_{z}}{2 D}\right)^{2} \omega^{2}
$$

which results in $k_{R M_{1}}$ tending to $k_{s}=\omega \sqrt{\frac{m_{s}}{G^{*} h}}$ with $k_{s}$, the corrected shear wavenumber.

- $k_{R M_{2}}$ is evanescent until the cut-on frequency given by Eq. (9) after which it becomes propagating and reaches the membrane wavenumber $k_{m}=\omega \sqrt{\frac{I_{z}}{D}}$ at high frequency.

$-k_{s}$ is always greater than $k_{m}$ since $\frac{k_{s}}{k_{m}}=\sqrt{\frac{2}{\kappa(1-\nu)}}>1$ 
Based on the above observations, Eq. (7) is rewritten in a compact form as,

$$
k_{R M_{1,2}}=\sqrt{\frac{1}{2}\left[k_{s}^{2}+k_{m}^{2} \pm \sqrt{4 k_{b}^{4}+\left(k_{s}^{2}-k_{m}^{2}\right)^{2}}\right]} .
$$

The cut-on frequency can be obtained by considering $k_{R M_{2}}=0$, that is,

$$
k_{s}^{2}+k_{m}^{2}=\sqrt{4 k_{b}^{4}+\left(k_{s}^{2}-k_{m}^{2}\right)^{2}} \Rightarrow k_{b}^{4}=k_{s}^{2} k_{m}^{2}
$$

and thus

$$
f_{\text {cut-on }}=\frac{1}{2 \pi} \sqrt{\frac{G^{*} h}{I_{z}}} .
$$

Further, it is observed that the high frequency asymptote given by Ghinet and Atalla [27] as

$$
k_{G \& A}=\omega \sqrt{\frac{4 I_{z} m_{s}}{G^{*} h I_{z}+m_{s} D}} .
$$

differs from the correct estimation of the high frequency asymptote $k_{s}$. It may be noted that Eq. (5) can be obtained from two of the three equilibrium equations (derived by a Newtonian approach) for thick plates [46]. For the sake of completeness, the dispersion relation resulting from third equilibrium equation [27] is presented here. The natural wavenumber from the third equilibrium equation is given by,

$$
k_{p}=k_{R M_{3}}=\sqrt{\frac{2}{1-\nu} \frac{I_{z} \omega^{2}-G^{*} h}{D}}=\sqrt{\delta_{s}^{2}-\frac{2}{1-\nu} \frac{k_{b}^{4}}{k_{s}^{2}}}
$$

where $\delta_{s}=\omega \sqrt{\frac{\rho}{G}}$ is the pure shear wavenumber of the isotropic elastic layer. Similar to $k_{R M_{2}}, k_{R M_{3}}$ is also evanescent until a cut-on frequency $\left(f_{\text {cut-on }}\right)$ but reaches the asymptote $\delta_{s}$ at higher frequencies as

$$
\delta_{s}^{2} \gg \frac{2}{1-\nu} \frac{k_{b}^{4}}{k_{s}^{2}}
$$

when $\omega \rightarrow+\infty$. Fig. 3 illustrates these asymptotic behaviours of the solutions of $k_{R M}$ for a plasterboard of thickness $50 \mathrm{~mm}$. Mechanical properties of the materials used in this paper can be obtained from Table 1.

The transmission loss across an infinite layer surrounded by air (defined by the equation $\left.V_{M}=[T]_{2 \times 2} V_{M^{\prime}}\right)$ for an oblique incidence may be computed from the following expression:

$$
\mathrm{TL}=-10 \log _{10} \tau
$$


Table 1: Material properties of few typical elastic isotropic layers used in this paper

\begin{tabular}{ccccc}
\hline Properties & Aluminium & Plasterboard & Concrete & Soft layer \\
\hline$\rho\left(\mathrm{kg} / \mathrm{m}^{3}\right)$ & 2780 & 700 & 2150 & 8 \\
$E(\mathrm{GPa})$ & 71 & 3 & 33 & 0.00016 \\
$\eta$ & 0.01 & 0.08 & 0.1 & 0.1 \\
$\nu$ & 0.3 & 0.22 & 0.23 & 0.44 \\
\hline
\end{tabular}

where

$$
\tau(\theta)=4 /\left|T_{11}+T_{22}-\left(\frac{T_{12} \cos \theta}{Z_{0}}+\frac{T_{21} Z_{0}}{\cos \theta}\right)\right|^{2}
$$

is the transmission factor, $Z_{0}=\rho_{0} c_{0}$ is the characteristic impedance of air and $\rho_{0}$ is the density of air. In this paper, the values for $c_{0}$ and $\rho_{0}$ are taken as $343 \mathrm{~m} . \mathrm{s}^{-1}$ and $1.2 \mathrm{~kg} . \mathrm{m}^{-3}$, respectively. The reader may note that for plate theories, the transmission factor is reduced to the form

$$
\tau(\theta)=1 /\left|1-\frac{Z_{P} \cos \theta}{2 Z_{0}}\right|^{2} .
$$

In case of a diffuse field excitation with minimum and maximum angle of incidences as $\theta_{\min }$ and $\theta_{\max }$, respectively, the TL is obtained computing the following integral:

$$
\mathrm{TL}_{\mathrm{d}}=-10 \log _{10}\left[\frac{\int_{\theta_{\min }}^{\theta_{\max }} \tau(\theta) \sin \theta \cos \theta \mathrm{d} \theta}{\int_{\theta_{\min }}^{\theta_{\max }} \sin \theta \cos \theta \mathrm{d} \theta}\right] .
$$

TL computed based on both thin and thick plate theories for the materials given in Table 1, are analyzed in the Section 5.

It must be realized that both thin and thick plate theories neglect the compressional mode (also called the symmetric or dilatational mode) and allow only anti-symmetric modes (i.e, bending and/or shear modes), since the plate velocity is assumed to be constant through the thickness direction.

\subsection{Wave propagation in elastic isotropic solids}

From the principles of the theory of elasticity, it can be derived that two fundamental waves can propagate through an isotropic medium corresponding to longitudinal and shear displacement in the solid. Longitudinal $\left(\delta_{l}\right)$ 
and shear $\left(\delta_{s}\right)$ wave numbers are given as [42],

$$
\delta_{l}=\omega \sqrt{\frac{\rho}{\lambda+2 \mu}}=\omega \sqrt{\frac{\rho}{K}}, \quad \delta_{s}=\omega \sqrt{\frac{\rho}{\mu}},
$$

where $K=\lambda+2 \mu$ is the compressional modulus of the elastic solid which highly influences the symmetric motions of the layers in a solid,

$$
\lambda=\frac{E \nu}{(1+\nu)(1-2 \nu)}
$$

is the first Lamé coefficient and $\mu=G$ is the second Lamé coefficient.

Assuming an acoustic wave incident on the surface of the solid with angle $\theta$, the resulting wavenumbers that propagate inside the elastic solid have a $z$ component of the form,

$$
k_{l z}=\sqrt{\delta_{l}^{2}-k_{t}^{2}}, \quad k_{s z}=\sqrt{\delta_{s}^{2}-k_{t}^{2}}
$$

By following Folds and Loggins [47], the state vector is taken as $V^{E S}=$ $\left\{u, v, \sigma_{z z}, \sigma_{x z}\right\}^{T}$ where the superscript ES denotes elastic solid, $\sigma_{x z}$ and $u$ are the transverse components of the stress and the velocity, and $\sigma_{z z}$ and $v$ the normal components of the stress and the velocity respectively. The transfer matrix for elastic solid is then expressed as

$$
V_{M}^{E S}=\left[T^{E S}\right]_{4 \times 4} V_{M^{\prime}}^{E S}
$$

where the elements of the matrix $\left[T^{E S}\right]$ are given in the Appendix A. For the computation of sound transmission loss across an infinite layer based on the solid transfer matrix, the reader may refer to the procedure given in the book by Allard and Atalla [42].

Based on the type of backing at point $M^{\prime}$, the frequency of the first compressional mode of an elastic layer is given as

$$
f_{\text {comp }}=\frac{1}{\gamma h} \sqrt{\frac{K}{\rho}},
$$

where $\gamma$ takes on the values 2 (half wave frequency) and 4 (quarter wave frequency) for anechoic and rigid backing respectively.

Since the motion both of the anti-symmetric and compressional mode of an infinite layer of finite thickness can be expressed based on the theory of elasticity, calculations of the transfer matrix for elastic solids are considered here as reference to the analyse using plate theories. By referring to the two fundamental wavenumbers $\left(\delta_{l}\right.$ and $\left.\delta_{s}\right)$ of the elastic isotropic solid, it can be understood that the bending wavenumber is a complex combination of these fundamental wavenumbers. It is, however, not straightforward to see this relation from the above equations for elastic isotropic solids. 


\section{Comparison between thin and thick plate theories}

\subsection{Frequency limit of thin plate theory in comparison with thick plate theory}

In this section, natural propagating wavenumbers of thin and thick plate theories are used to find the frequency limit of the thin plate theory. From the Fig. 3 , it can be seen that the thick plate wavenumber $\left(k_{R M_{1}}\right)$ clearly deviates from the bending wavenumber $\left(k_{b}\right)$ after certain frequency. It may be noted that, though there are totally three outgoing waves characterized by wavenumbers $\left(k_{R M_{1}}, k_{R M_{2}} \& k_{R M_{3}}\right), k_{R M_{1}}$ is considered for the present analysis as it is the only wavenumber that is always propagative. Additionally, since the deviation between $k_{R M_{1}}$ and $k_{b}$ starts well before the cut-on frequency $\left(f_{\text {cut-on }}\right), k_{R M_{1}}$ would be appropriate to derive the frequency limit of thin plate theory. By defining $C_{k}=\frac{k_{b}}{k_{s}}$, the ratio between bending and shear wavenumbers, error percentage $(\epsilon)$ between the propagating wavenumbers of the thin and thick plate theories is expressed as

$$
\epsilon=\left(1-\frac{1}{k_{R M_{1}} / k_{b}}\right) 100 \%
$$

where

$$
\frac{k_{R M_{1}}}{k_{b}}=\frac{1}{2} \sqrt{\frac{2+\kappa(1-\nu)}{C_{k}^{2}}+\sqrt{16+\left[\frac{2-\kappa(1-\nu)}{C_{k}^{2}}\right]^{2}}} .
$$

The thin plate theory will be valid while $k_{s}$ is negligible compared to $k_{b}$ $\left(k_{b}>>k_{s}\right)$. The value for $C_{k}$ can be chosen such that $\epsilon$ is below an accepted error percentage and the frequency range of validity for thin isotropic plate can be expressed as given by Eq. (20).

$$
k_{b} \geq C_{k} k_{s} \Rightarrow f \leq f_{\text {thin } / \text { thick }}=\frac{G^{*} h}{2 \pi C_{k}^{2}} \sqrt{\frac{1}{m_{s} D}}=\frac{\kappa}{4 \pi h C_{k}^{2}} \sqrt{\frac{12 E}{\rho} \frac{1-\nu}{1+\nu}}
$$

where $f_{\text {thin/thick }}$ is the frequency limit of the 'thin' plate theory by keeping the 'thick' plate theory as reference. For instance, choosing $C_{k}=4$ for typical isotropic layer corresponds to an error percentage $(\epsilon)$ around $2 \%$ between $k_{R M_{1}}$ and $k_{b}$.

\subsection{Coincidence and critical frequencies of thick plate}

As discussed in the earlier sections, thin plate theory allows only bending waves to propagate in the elastic plate and shear wave propagation is included by thick plate theory to correctly capture the anti-symmetric motion of the plate. Due to this additional anti-symmetric motion in the plate, the 
coincidence and critical frequency expressions obtained from thin plate theory need to be rewritten with terms corresponding to shear and rotational inertia.

The coincidence frequency between a plate and an acoustic wave incident on the plate at an angle $\theta$ is defined as the frequency at which the transverse component of the incident wavenumber is equal to the natural propagating wavenumber of the plate. In the case of thin plates, the natural propagating wavenumber is the bending wavenumber and the coincidence frequency is expressed as,

$$
k_{b}=k_{0} \sin \theta \Longrightarrow f_{\text {coinc }_{\text {thin }}}=\frac{1}{2 \pi}\left(\frac{c_{0}}{\sin \theta}\right)^{2} \sqrt{\frac{m_{s}}{D}} .
$$

For thick plates, as the natural propagating wavenumber is given by $k_{R M_{1}}$, the coincidence frequency is expressed as,

$$
k_{R M_{1}}=k_{0} \sin \theta \Longrightarrow f_{\text {coinc }_{\text {thick }}}=\frac{\left(c_{0} / \sin \theta\right)^{2}}{2 \pi \sqrt{\left(\frac{D}{m_{s}}-\frac{c_{0}^{2}}{\sin ^{2} \theta} \frac{I_{z}}{m_{s}}\right)\left(1-\frac{c_{0}^{2}}{\sin ^{2} \theta} \frac{m_{s}}{G^{*} h}\right)}} .
$$

In case of diffuse field excitation, the elastic layer is subjected to all coincidence frequencies corresponding to $\theta=[0, \pi / 2]$ and the lowest coincidence frequency is called the critical frequency. In other words, it is the frequency at which the speed of sound is equal to the speed of natural propagating waves of the plate. This can be computed by letting $\sin \theta=1$ in the coincidence frequency expression. The critical frequency obtained from thin plate theory is given by $k_{b}=k_{0}$, that is,

$$
f_{\text {cri }_{\text {thin }}}=\frac{c_{0}^{2}}{2 \pi} \sqrt{\frac{m_{s}}{D}} .
$$

From the Eq. (22), the critical frequency for thick plate is obtained from $k_{R M_{1}}=k_{0}$, that is,

$$
f_{\text {cri }_{\text {thick }}}=\frac{c_{0}^{2}}{2 \pi \sqrt{\left(\frac{D}{m_{s}}-c_{0}^{2} \frac{I_{z}}{m_{s}}\right)\left(1-c_{0}^{2} \frac{m_{s}}{G^{*} h}\right)}} .
$$

It may be noted that the Eqs. (22) and (24) tend to coincidence and critical frequencies obtained from thin plate theory as $I_{z} \rightarrow 0$ and $G^{*} \rightarrow \infty$. As an illustration, for $12.5 \mathrm{~mm}$ plasterboard, the coincidence frequencies computed 


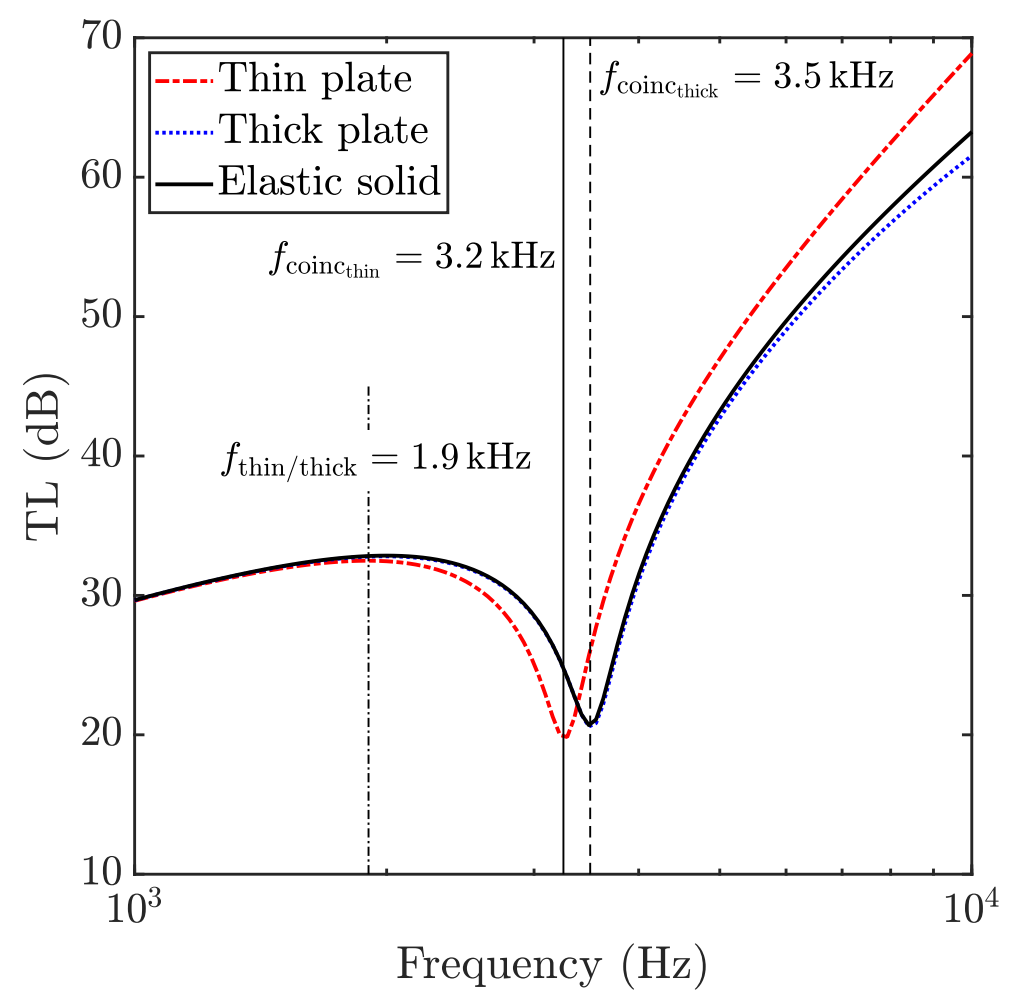

Figure 4: Transmission loss for the infinitely extent plasterboard of thickness $12.5 \mathrm{~mm}$ (properties are mentioned in Table 1) under plane wave excitation with $\theta=60^{\circ}$ with coincidence frequencies computed from thin \& thick theories and it can be observed that the estimation of coincidence frequency from thick plate theory is in good agreement with theory of elasticity computation.

from both plate theories are indicated in the Fig. 4. It is observed from the Fig. 4 that the coincidence frequency computed from Eq. (22) is in good agreement with elasticity theory. Therefore, it is worth noting that Eqs. (21) and (23) are indeed limited to thin plates only, where the transition from thin to thick plates is given by the frequency $f_{\text {thin/thick }}$ in Eq. (20).

\section{Frequency limit of plate theories in comparison with theory of elasticity}

When the thickness of the layer is small compared to the lateral dimension and the longitudinal wavelength $\left(\lambda_{l}=2 \pi / \delta_{l}\right)$ is large compared to thickness, a plate theory, controlled by the anti-symmetric motion, is generally considered. On the contrary, when the thickness of the plate is of the order 
of magnitude of longitudinal wavelength, due to the variation in velocity through the thickness of the layer, both symmetric and anti-symmetric motions contribute for the resulting motion of the plate after a certain frequency (Fig. 5). This implies that plate theories might not be able to predict the

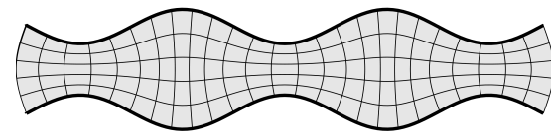

(a) Symmetric mode

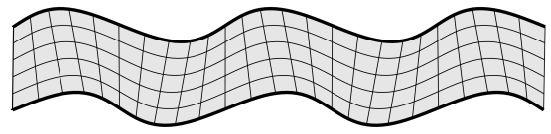

(b) Anti-symmetric mode

Figure 5: Vibrating modes of an infinitely extent elastic layer. While the symmetric mode corresponds to the thickness stretch motion of the layer where the particle velocity varies through the thickness, the anti-symmetric motion corresponds to the bending and shear motions of the layer where the particle velocity is constant through the thickness.

correct vibro-acoustic behaviour of the elastic layer after this frequency as they assume only anti-symmetric motions in the plate. Therefore, finding this frequency limit of plate theories is necessary and in this section, based on the symmetric and anti-symmetric motions of the plate, the analytical expression of the frequency limit of plate theories is derived.

Contributions of symmetric and anti-symmetric motions of an isotropic layer can be quantified by the impedances or admittances, by following Dym and Lang [48]. Impedances of symmetric and anti-symmetric motions are defined as follows [48],

$$
\begin{aligned}
& Z_{s}=2 \frac{p_{M}+p_{M^{\prime}}}{v_{M}-v_{M^{\prime}}}, \\
& Z_{a}=2 \frac{p_{M}-p_{M^{\prime}}}{v_{M}+v_{M^{\prime}}},
\end{aligned}
$$

where $Z_{s}$ and $Z_{a}$ are symmetric, anti-symmetric impedances of the layer respectively and $p$ and $v$ are pressure and velocity respectively. It may be noted that though Dym and Lang [48] assumed $p_{M^{\prime}}=0$ in their analysis, later, they have corrected the definitions of impedances with non-zero pressure values [49].

The above equations are rewritten to obtain the transfer matrix relations as follows,

$$
\left(\begin{array}{l}
p \\
v
\end{array}\right)_{M}=\frac{1}{Y_{a}-Y_{s}}\left[\begin{array}{cc}
Y_{a}+Y_{s} & 1 \\
4 Y_{a} Y_{s} & Y_{a}+Y_{s}
\end{array}\right]\left(\begin{array}{l}
p \\
v
\end{array}\right)_{M^{\prime}}
$$

Here, $Y_{s}=1 / Z_{s}$ and $Y_{a}=1 / Z_{a}$ are the symmetric and anti-symmetric admittances of the layer respectively. It can be checked that, when the antisymmetric admittance is larger than the symmetric admittance (or $Y_{a} \gg Y_{s}$ ), the transfer matrix in Eq. (27) reduces to the transfer matrix of the plate 
given by Eq. (2). Thus the ratio between $Y_{a}$ and $Y_{s}$ could be a good criterion to obtain the frequency limit of plate theories. Comparing the longitudinal wavelength $\left(\lambda_{l}\right)$ to the thickness of the plate seems less accurate as it does not compare the symmetric motion to the anti-symmetric motion.

Since the symmetric motion is controlled by the longitudinal wave of the layer, the transfer matrix from Eq. (16) is deduced at normal incidence as

$$
\left(\begin{array}{l}
p \\
v
\end{array}\right)_{M}=\left[\begin{array}{cc}
\cos h \delta_{l} & \frac{\mathrm{j} \omega \rho}{\delta_{l}} \sin h \delta_{l} \\
\frac{\mathrm{j} \delta_{l}}{\omega \rho} \sin h \delta_{l} & \cos h \delta_{l}
\end{array}\right]\left(\begin{array}{l}
p \\
v
\end{array}\right)_{M^{\prime}} .
$$

By equating the above equation with Eq. (27), the symmetric admittance is obtained as

$$
Y_{s}=\frac{h \delta_{l}\left(\cos h \delta_{l}-1\right)}{2 \mathrm{j} m_{s} \omega \sin h \delta_{l}}=-\frac{h \delta_{l}}{2 \mathrm{j} m_{s} \omega} \tan \frac{h \delta_{l}}{2} .
$$

Approximating the tangent function by a Taylor series expansion (up to first order), the symmetric admittance can be written as,

$$
\tan \frac{h \delta_{l}}{2} \approx \frac{h \delta_{l}}{2} \Longrightarrow Y_{s} \approx \widetilde{Y}_{s}=-\frac{\left(h \delta_{l} / 2\right)^{2}}{\mathrm{j} \omega m_{s}}=\frac{\mathrm{j} \omega h}{4 K}
$$

Since the anti-symmetric motion is controlled by the transverse wavenumber of the incident wave and plate theories capture this type of motion, antisymmetric admittance is computed from plate theories as given by Eq. (3).

The minimum value of the absolute ratio between the anti-symmetric and symmetric admittance, denoted by $C_{y}$, is used to find the frequency limit of plate theories. Expressing the anti-symmetric admittance $\left(Y_{a}\right)$ from thin plate theory and the symmetric admittance $\left(\widetilde{Y}_{s}\right)$ from Eq. (30), the frequency limit of plate theories is expressed as,

$$
\left|\frac{Y_{a}}{\widetilde{Y}_{s}}\right| \geq C_{y} \Rightarrow f \leq f_{\text {plate }_{\text {solid }} \text { oi }}=\frac{c_{0}^{2}}{2 \pi \sin ^{2} \theta} \sqrt{\frac{m_{s}}{2 D} \pm \sqrt{\left(\frac{m_{s}}{2 D}\right)^{2} \pm \frac{4 K}{h C_{y} D} \frac{\sin ^{4} \theta}{c_{0}^{4}}}}
$$

The above expression is valid for oblique incidence whereas in case of diffuse field excitation, the following expression may be used to compute the frequency limit of plate theories.

$$
\left|\frac{Y_{a}}{\widetilde{Y}_{s}}\right| \geq C_{y} \Rightarrow f \leq f_{\text {plate }_{\text {solid }} \text { df }}=\frac{c_{0}^{2}}{2 \pi} \sqrt{\frac{m_{s}}{2 D} \pm \sqrt{\left(\frac{m_{s}}{2 D}\right)^{2} \pm \frac{4 K}{h C_{y} D} \frac{1}{c_{0}^{4}}}} .
$$


The above frequency limits are computed by keeping the loss factor $(\eta)$ to be zero. The subscript 'plate/solid' in the above equations means that the frequency limit is for 'plate' theories in general (as even higher order plate theories also do not account for symmetric motion) by keeping as a reference the theory of 'elastic solids'. Further, the sub-subscripts 'oi' and 'df' correspond to 'oblique incidence' and 'diffuse field' respectively. It may be observed that the relation $\left|\frac{Y_{a}}{\widetilde{Y}_{s}}\right| \geq C_{y}$ yields four positive roots for the frequency. Out of these four roots, only the minimum of pure real roots is considered for $f_{\text {plate/solid }_{\mathrm{oi}}}$ and $f_{\text {plate/solid }_{\mathrm{df}}}$. It may also be noted that the expression for $f_{\text {plate/solid }}$ can be modified in two ways. First, by including higher order terms for the tangent function to get $\widetilde{Y}_{s}$. Second, by using the anti-symmetric mechanical admittance from thick plate theory. Though these two ways might improve the frequency limit, the final expression for $f_{\text {plate/solid }}$ df would become more complex. Further, as discussed in the next section, the frequency limits given by Eqs. (31) and (32) are sufficient enough for typical single layer walls used in industry. A concrete layer of $140 \mathrm{~mm}$ is taken to illustrate the nature of the symmetric and anti-symmetric admittances of the elastic layer and presented in the Fig. 6 .

From the Fig. 6, it can be seen that the anti-symmetric admittance is larger compared to the symmetric admittance at low frequency range. The symmetric admittance is seen to become of the same order of magnitude or larger compared to the anti-symmetric impedance at around $2000 \mathrm{~Hz}$. By letting the factor $C_{y}$ to be 10, the frequency limit of plate theories is computed from Eq. (32). This means that the anti-symmetric admittance is one order of magnitude larger than the symmetric admittance and from this frequency onwards use of plate theories is not recommended to compute the acoustic indicators. Therefore, it is advisable to adopt the theory of elasticity for computations after this frequency limit. 


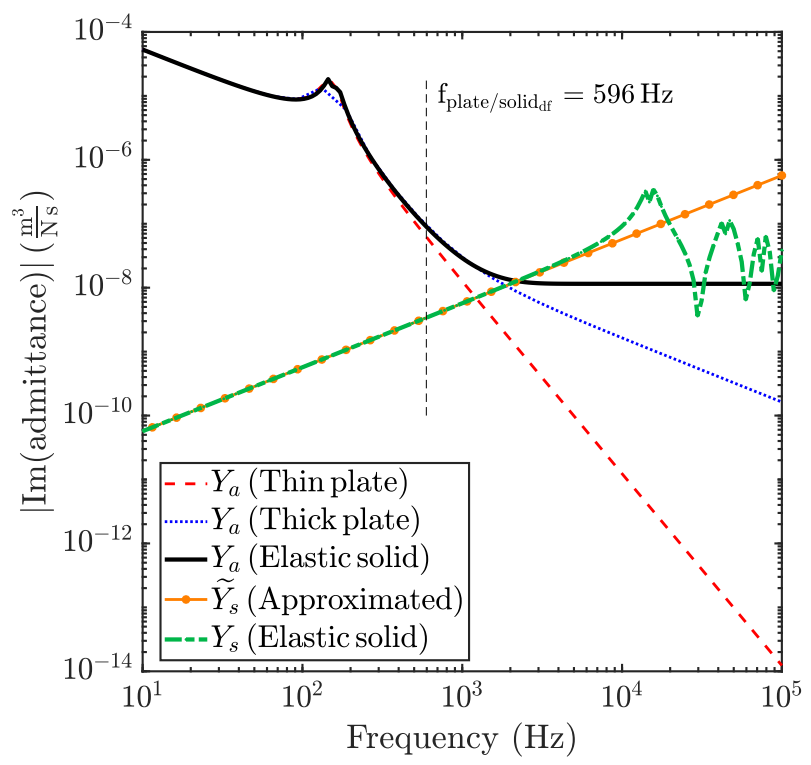

Figure 6: Admittances of a concrete layer of thickness $140 \mathrm{~mm}$ (properties are mentioned in Table 1) under plane wave excitation with $\theta=60^{\circ}$. It is observed that, from low frequencies till the limiting frequency $\left(f_{\text {plate/solid }}\right)$, the symmetric admittance is lesser compared to the anti-symmetric admittance which supports the applicability of plate theories till $f_{\text {plate } / \text { solid }_{\mathrm{df}}}$.

\section{Numerical examples}

In this section, transmission loss (TL) of different material layers (with properties listed in Table 1) are presented to illustrate the frequency limits obtained in sections 3 and 4 . Both, oblique plane wave incidence of $60^{\circ}$ and diffuse fields, are used to compute TL. Since $f_{\text {plate/solid }}$ is the minimum of all the possible coincidence frequencies obtained from $f_{\text {plate/solid }_{\mathrm{oi}}}$, in this section, $f_{\text {plate/solid }}$ is indicated as the limit of plate theory. Though elasticity theory is considered as reference to analyse the plate theories, additional validation from finite element method (FEM) is also presented in some of the TL plots in this section.

FEM simulations are computed using Comsol Multiphysics@ software. The acoustical variables (pressure, velocity fileds...) are computed in the coupled system (PML-air-material-air-PML) using the "Comsol Pressure Acoustics" interface (Helmholtz equation) for air and "Structural Mechanics branch" for the material (elastic material in "Solid Mechanic"). The interface between air and the material is modelized using "fluid-structure interface". The dimensions of each material are $60 \mathrm{~cm} \times 60 \mathrm{~cm}$ (the thickness is the real thick- 
ness) and periodic lateral conditions are chosen. The domain is adjusted (in particular the dimensions of the air domains) and meshed with respect to a 10 elements per wavelength (of the incident plane wave excitation) criterion based on the maximal frequency. For example, at $125 \mathrm{~Hz}$, the number of resolved degrees of freedom is 444675, the complete mesh consists of 29889 domain elements, 8802 boundary elements and 812 edge elements.

In Figs. 7 and 8, TL computed from different theories (discussed in the section 2) are presented for comparison along with frequency limits expressed in sections 3 and 4 . It is observed from these plots that, for material like aluminium (with typical value of thickness used in industries), the thin plate theory would be sufficient to model the vibro-acoustic behaviours as both $f_{\text {thin/thick }}$ and $f_{\text {plate/solid }}$ are spotted near the maximum audible frequency.

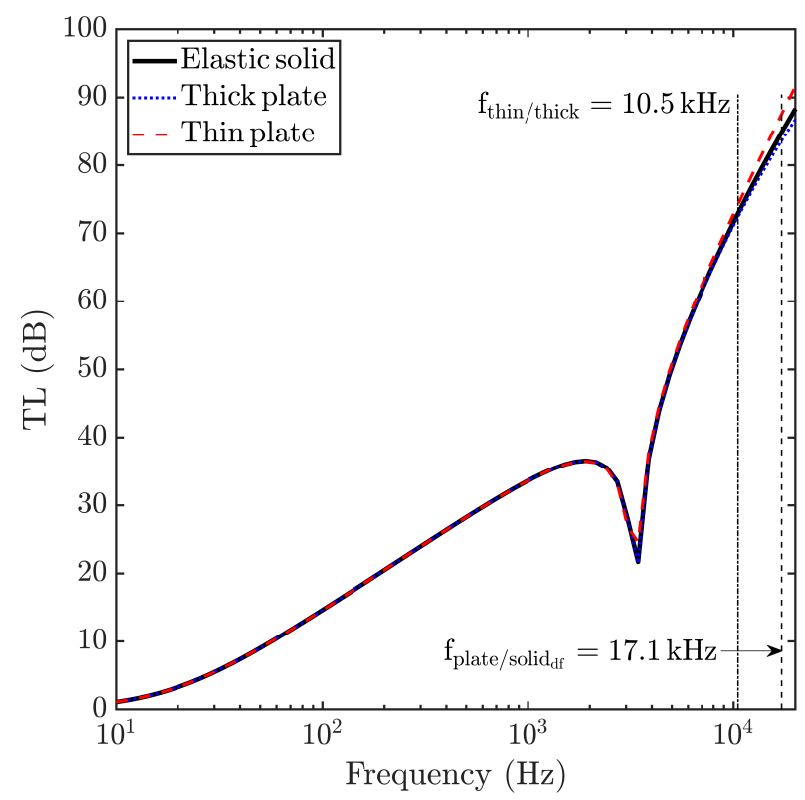

Figure 7: Transmission loss for an aluminium layer of thickness $5 \mathrm{~mm}$ (properties mentioned in Table 1) under plane wave excitation with $\theta=60^{\circ}$. It is seen that thin plate theory is adequate to compute the vibro-acoustic indicators as both limiting frequencies $\left(f_{\text {thin/thick }} \& f_{\text {plate/solid }_{\text {df }}}\right)$ are in the high frequencies. 


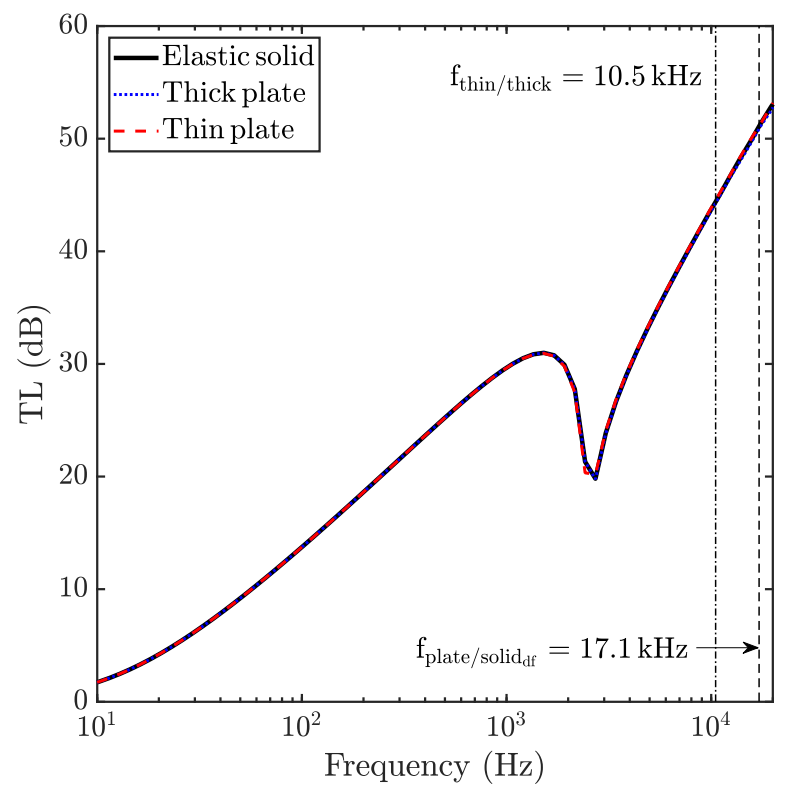

Figure 8: Transmission loss for an aluminium layer of thickness $5 \mathrm{~mm}$ (properties mentioned in Table 1) under diffuse field excitation. It is seen that thin plate theory is adequate to compute the vibro-acoustic indicators as both limiting frequencies $\left(f_{\text {thin/thick }}\right.$ $\left.\& f_{\text {plate/solid }}\right)$ are in the high frequencies.

For the plasterboard of $12.5 \mathrm{~mm}$, it is seen from the Figs. 9 and 10 that TL computed from thin plate theory is begining to deviate from the elasticity theory computation whereas thick plate theory is still in good agreement with the elasticity theory until the limiting frequency $f_{\text {plate/solid }}$. This explains the need to include the effect of shear into the anti-symmetric motion via thick plate theory. Therefore, for these kind of materials, thick plate theory would be appropriate to compute the acoustic indicators. 


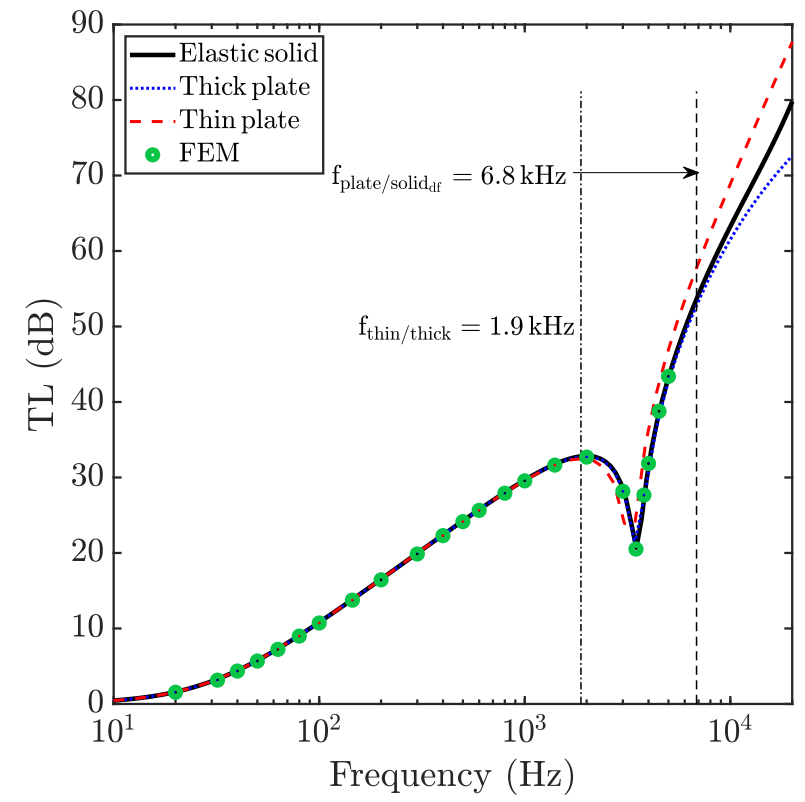

Figure 9: Transmission loss for a plasterboard layer of thickness $12.5 \mathrm{~mm}$ (properties mentioned in Table 1) under plane wave excitation with $\theta=60^{\circ}$. Deviations of thin and thick plate theories from the elastic solid theory (or FEM) are observed after $f_{\text {thin/thick }}$ and $f_{\text {plate/solid }}$ respectively.

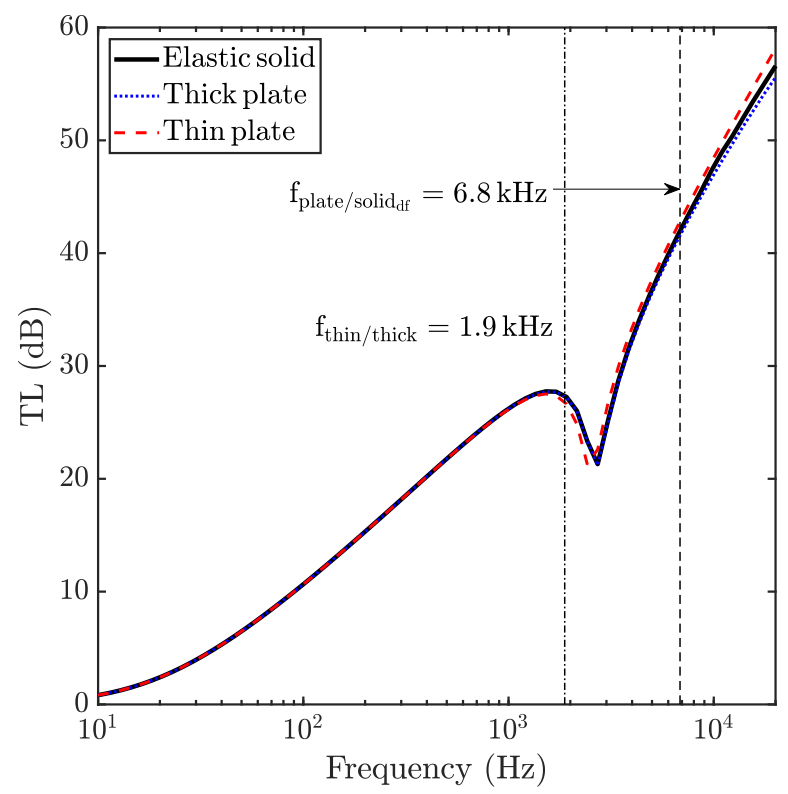

Figure 10: Transmission loss for a plasterboard layer of thickness $12.5 \mathrm{~mm}$ (properties mentioned in Table 1) under diffuse field excitation. Deviations of thin and thick plate theories from the elastic solid theory are observed after $f_{\text {thin/thick }}$ and $f_{\text {plate/solid }}$ respectively. 
In case of a concrete layer with $140 \mathrm{~mm}$ thickness, it is noted from Figs. 11 and 12 that a similar trend is observed as for the plasterboard, that is, thin and thick plate theories are starting to deviate from the elasticity theory computation at frequencies above $f_{\text {thin/thick }}$ and $f_{\text {plate/solid }}$, respectively. One might also observe two notable points from the TL plots of concrete and plasterboard. First, the coincidence frequency occurs after the limit frequency of thin plate $\left(f_{\text {thin/thick }}\right)$ in plasterboard whereas it can be spotted before $f_{\text {thin/thick }}$ in concrete. This implies that even for thicker material the thin plate theory might be still valid after the coincidence frequency. The second notable point is that the symmetric motion (or compressional motion) effect clearly appears in concrete. In Fig. 12, the second minima in the TL computed from the theory of elasticity corresponds to the compressional frequency $\left(f_{\text {comp }} \approx 15 \mathrm{kHz}\right.$ ) given by Eq. (17). Therefore, it is inferred that the compressional mode can still be neglected for plasterboard whereas it has to be taken into account for the concrete layer and this is possible via employing the theory of elasticity. The same is observed from the TL plots (Figs. 13 \& 14) of soft layer with $20 \mathrm{~mm}$ thickness. It can be seen that TL of soft layer is greatly influenced by the symmetric motion after the frequency limit $f_{\text {plate/solid }}$.

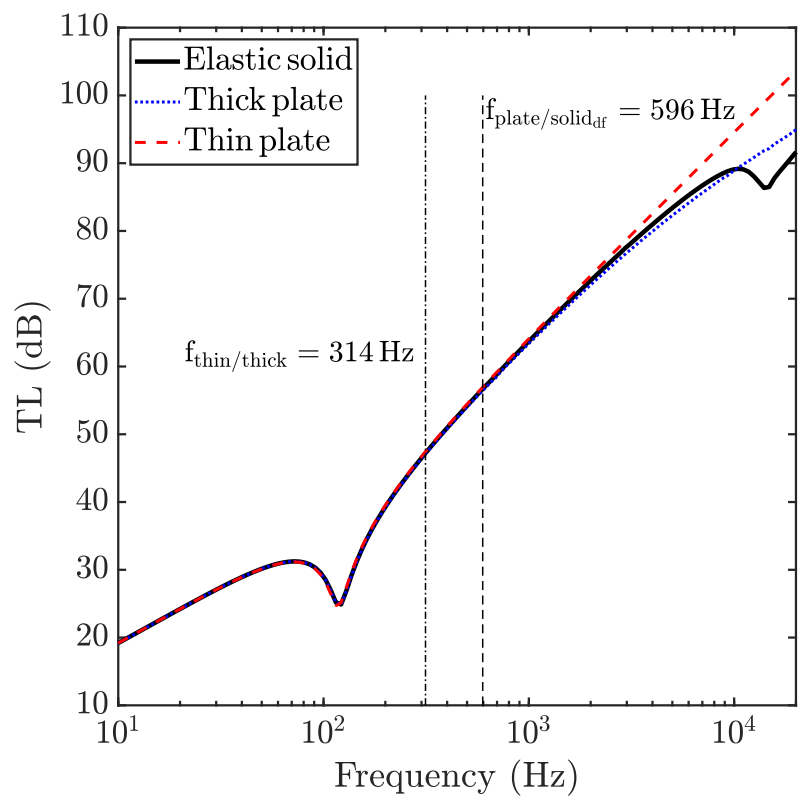

Figure 12: Transmission loss for a concrete layer of thickness $140 \mathrm{~mm}$ (properties mentioned in Table 1) under diffuse field excitation. Deviations of thin and thick plate theories from the elastic solid theory start to appear after $f_{\text {thin/thick }}$ and $f_{\text {plate/solid }}$ respectively. 


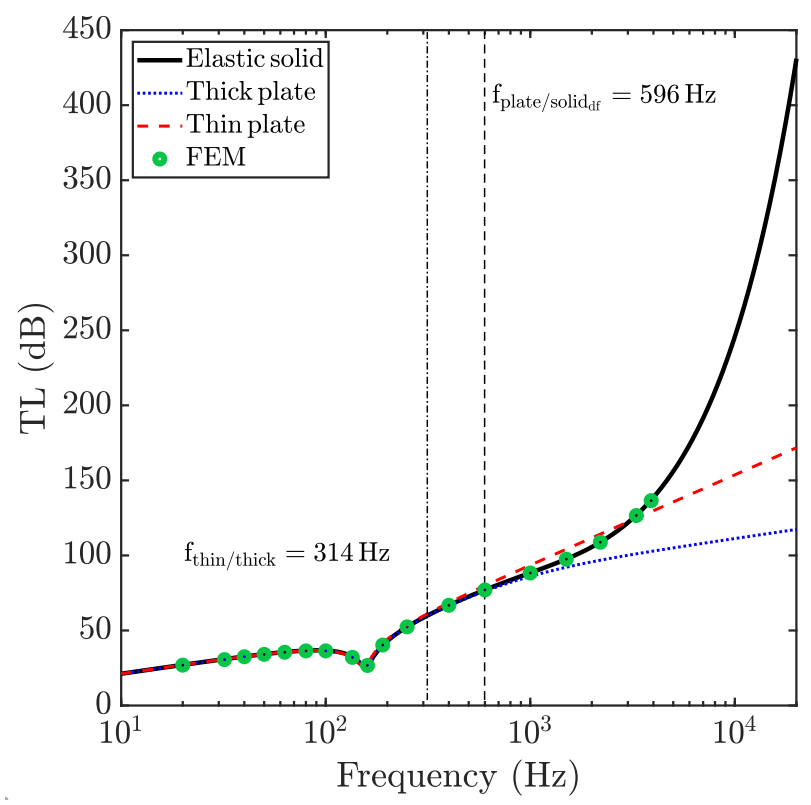

Figure 11: Transmission loss for a concrete layer of thickness $140 \mathrm{~mm}$ (properties mentioned in Table 1) under plane wave excitation with $\theta=60^{\circ}$. Deviations of thin and thick plate theories from the elastic solid theory (or FEM) start to appear after $f_{\text {thin/thick }}$ and $f_{\text {plate/solid }}$ rf respectively.

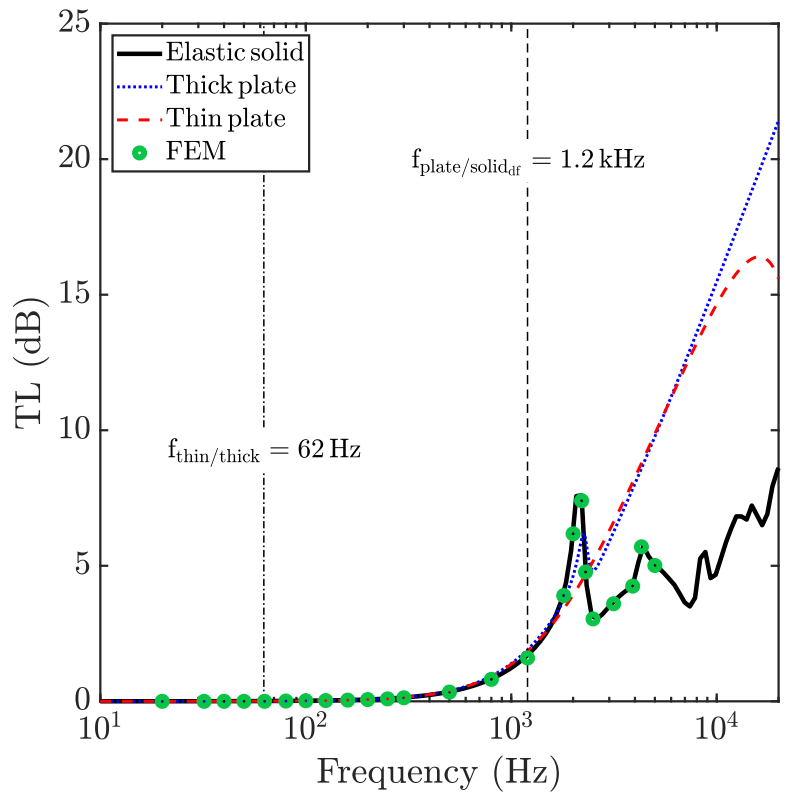

Figure 13: Transmission loss for a soft layer of thickness $20 \mathrm{~mm}$ (properties mentioned in Table 1) under plane wave excitation with $\theta=60^{\circ}$. Deviations of plate theories from the elastic solid theory (or FEM) start to appear after $f_{\text {plate/solid }}$. 


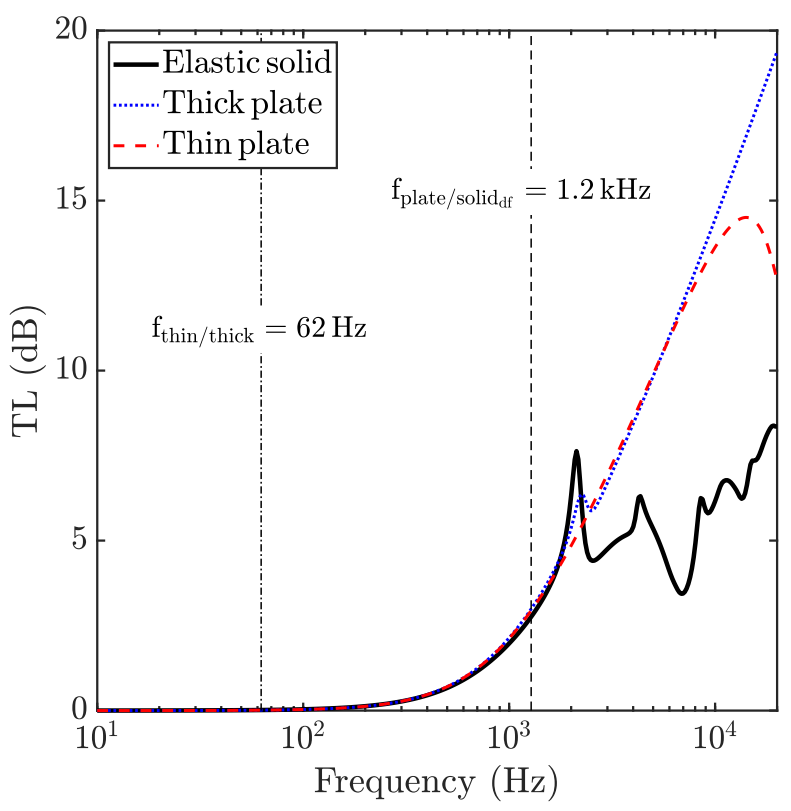

Figure 14: Transmission loss for a soft layer of thickness $20 \mathrm{~mm}$ (properties mentioned in Table 1) under diffuse field excitation. Deviations of plate theories from the elastic solid theory start to appear after $f_{\text {plate/solid }}$.

One can also observe from Eq. (20) that, for different materials of infinitely extending layers with same thickness, the frequency limit $f_{\text {thin/thick }}$ would result in different values despite thicknesses being the same. Therefore, it can be inferred that the use of thin plate theory requires proper combination of thickness and material properties (as given by Eq. (20)) rather than comparing the thickness to the lateral dimensions. Similar argument holds for $f_{\text {plate/solid }}$ as well. In the previous TL plots, the choice of the values of $C_{k}=4$ and $C_{y}=10$ are further confirmed by the TL variation of plate theories from the elasticity theory and the TL difference between the elasticity theory and plate theories are observed to be below $1 \mathrm{~dB}$ at the frequency limits. Of course, one can conveniently choose the appropriate value of $C_{k}$ and $C_{y}$ based on the tolerance accepted for the particular acoustic design.

\subsection{Further observation}

In the case of finite sized plates, generally, the acoustic indicators computed from the infinitely extent layer theories would yield some discrepancies in the low to mid frequencies compared with experimental tests. Therefore, there are some works in the literature $[5,50]$ which focus on correcting the 
acoustic indicators by introducing correction factors that account for geometrical size effect.

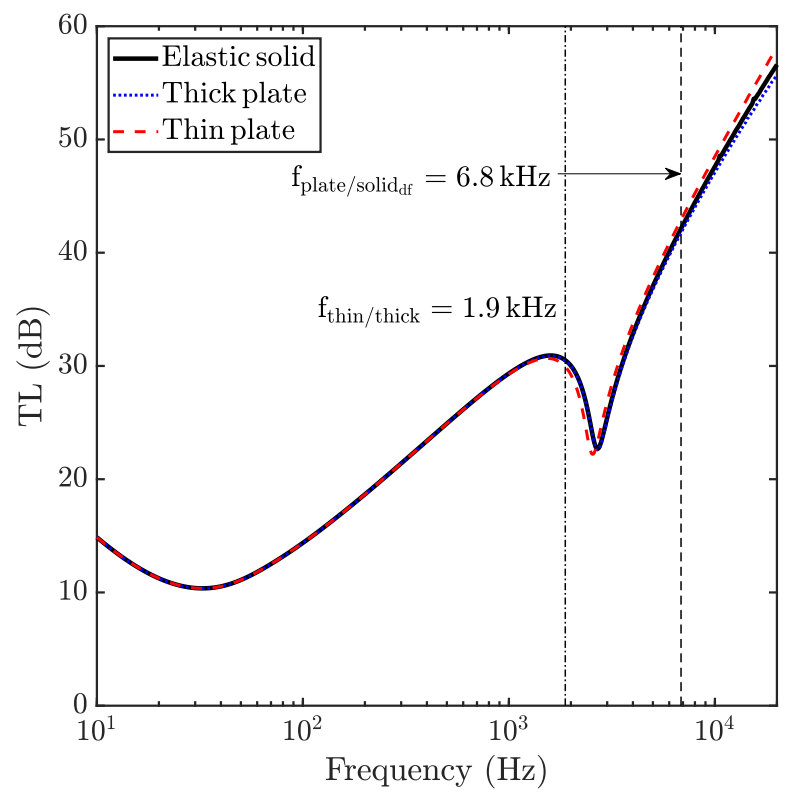

Figure 15: Transmission loss, computed from spatial windowing method by Rhazi and Atalla [50], across a finite size $(3 \mathrm{~m} \times 4 \mathrm{~m})$ plasterboard of thickness $12.5 \mathrm{~mm}$ (properties are mentioned in Table 1) under diffuse field excitation. It is observed that limiting frequencies computed for infinite plate are still valid for the finite plates as the size correction effects minimal near the critical frequency of the plate.

Since the radiation efficiency (which accounts for size correction in sound transmission problems) is reaching unity near the critical frequency of the plate [44], the effects due to finite size is mainly visible at low frequencies below the critical frequency. Since the frequency limits $\left(f_{\text {thin/thick }}\right.$ and $f_{\text {plate/solid }}$ ) of typical industrial materials fall near and/or after the critical frequency, these limiting expressions obtained from infinite plate theories are applicable to the finite size plate as well. For example, this can be observed from the transmission loss computed from the finite size correction model by Rhazi and Atalla [50] for the plasterboard of $12.5 \mathrm{~mm}$ thickness under diffuse field excitation in Fig 15.

\section{Concluding remarks}

The assumptions used in thin and thick plate theories limit their applicability in commonly used industrial materials after a certain frequency. Thin 
plate theory attains the limitation since it does not account for the shear effect in the anti-symmetric motion of the plate whereas this is considered in thick plate theories. Nevertheless, both types of plate theories are approximations since they neglect symmetric motion of the panel in their theoretical formulation. By analysing the wavenumbers and admittances of the investigated structures, two frequency limits were presented in this manuscript: 1) from the wave propagation analysis of the thick plate model, based on the Reissner-Mindlin plate hypothesis, the analytical expression for the limiting frequency of the thin plate model is derived, while 2) from comparing the symmetric and anti-symmetric admittances, an analytical expression for the limiting frequency of plate theories is derived. These two simple analytical expressions for computing the limit of thin and thick plate theories can be useful to choose the appropriate model in each case. Deviations of the TL predictions obtained from different models are observed above these two limiting frequencies. It is also shown that, although the limiting expressions are derived from infinite layer theories, they can be applied to finite sized layers as well. Due to the omission of shear effects in thin plate theories, the refined coincidence and critical frequencies are derived from thick plate theories. Finally, it is observed that plate theories quickly fail for materials that are too soft in terms of longitudinal compression.

\section{Acknowledgments}

The authors would like to gratefully acknowledge Marie-Skłodowska Curie Actions (MSCA) Project 765472 'N2N: No2Noise' for the financial support. 


\section{Appendix A. The transfer matrix of an elastic isotropic solid}

The transfer matrix of an elastic isotropic layer (defined by the equation $V^{E S}(M)=\left[T^{E S}\right]_{4 \times 4} V^{E S}\left(M^{\prime}\right)$ where $\left.V^{E S}=\left\{u, v, \sigma_{z z}, \sigma_{x z}\right\}^{T}\right)$ based on elasticity theory can be written as follows:

$$
\left[T^{E S}\right]_{4 \times 4}=\frac{1}{D_{1}+D_{2} k_{t}}\left[T_{p q}^{E S}\right] \quad \text { where } p, q=1 \text { to } 4 .
$$

The matrix elements $\left(T_{p q}^{E S}\right)$ are,

$T_{11}=T_{44}=D_{1} \cos \left(h k_{s z}\right)+D_{2} k_{t} \cos \left(h k_{l z}\right)$,

$T_{22}=T_{33}=D_{1} \cos \left(h k_{l z}\right)+D_{2} k_{t} \cos \left(h k_{s z}\right)$,

$T_{12}=T_{34}=-\mathrm{j}\left[D_{2} k_{l z} k_{s z} \sin \left(h k_{s z}\right)-D_{1} k_{t} \sin \left(h k_{l z}\right)\right] / k_{l z}$,

$T_{21}=T_{43}=\mathrm{j}\left[D_{2} k_{s z} k_{l z} \sin \left(h k_{l z}\right)-D_{1} k_{t} \sin \left(h k_{s z}\right)\right] / k_{s z}$,

$T_{13}=T_{24}=\omega k_{t}\left[\cos \left(h k_{s z}\right)-\cos \left(h k_{l z}\right)\right]$,

$T_{31}=T_{42}=\left(\frac{D_{1} D_{2}}{\omega^{2} k_{t}}\right) T_{13}$

$T_{14}=-\mathrm{j} \omega\left[k_{l z} k_{s z} \sin \left(h k_{s z}\right)+k_{t}^{2} \sin \left(h k_{l z}\right)\right] / k_{l z}$,

$T_{23}=-\mathrm{j} \omega\left[k_{l z} k_{s z} \sin \left(h k_{l z}\right)+k_{t}^{2} \sin \left(h k_{s z}\right)\right] / k_{s z}$,

$T_{32}=-\mathrm{j}\left[D_{2}^{2} k_{l z} k_{s z} \sin \left(h k_{s z}\right)+D_{1}^{2} \sin \left(h k_{l z}\right)\right] /\left(\omega k_{l z}\right)$,

$T_{41}=-\mathrm{j}\left[D_{2}^{2} k_{l z} k_{s z} \sin \left(h k_{l z}\right)+D_{1}^{2} \sin \left(h k_{s z}\right)\right] /\left(\omega k_{s z}\right)$,

where $D_{1}=\mu\left(k_{s z}^{2}-k_{t}^{2}\right)$ and $D_{2}=2 \mu k_{t}$. 


\section{References}

[1] L. Cremer, Theorie der schalldämmung dünner wände bei schrägem einfall, Akustische Zeitschrift 7 (1942) 81-104.

[2] M. Crocker, A. Price, Sound transmission using statistical energy analysis, Journal of Sound and Vibration 9 (1969) 469-486.

[3] E. Sewell, Transmission of reverberant sound through a single-leaf partition surrounded by an infinite rigid baffle, Journal of Sound and Vibration 12 (1970) 21-32.

[4] F. G. Leppington, K. Heron, E. G. Broadbent, S. M. Mead, Resonant and non-resonant acoustic properties of elastic panels. II. The transmission problem, Proceedings of the Royal Society of London. A. Mathematical and Physical Sciences 412 (1987) 309-337.

[5] M. Villot, C. Guigou, L. Gagliardini, Predicting the acoustical radiation of finite size multi-layered structures by applying spatial windowing on infinite structures, Journal of Sound and Vibration 245 (2001) 433-455.

[6] S. Ljunggren, A new type of solution for plate vibrations at low frequencies, Journal of Sound and Vibration 116 (1987) 125-136.

[7] A. E. H. Love, XVI. The small free vibrations and deformation of a thin elastic shell, Philosophical Transactions of the Royal Society of London.(A.) (1888) 491-546.

[8] J. L. Davy, Predicting the sound insulation of single leaf walls: Extension of Cremer's model, The Journal of the Acoustical Society of America 126 (2009) 1871-1877.

[9] M. Heckl, U. Donner, Schalldämmung dicker Wände (Sound insulation of thick walls), Rundfunktech Mitt 29 (1985) 287-291.

[10] R. Mindlin, Influence of rotary inertia and shear on flexural motions of isotropic elastic plates, ASME Journal of Applied Mechanics 18 (1951).

[11] E. Reissner, The effect of transverse shear deformation on the bending of elastic plates, ASME Journal of Applied Mechanics 12 (1945) A69-A77.

[12] H. Hencky, über die Berücksichtigung der Schubverzerrung in ebenen Platten, ingenieur-archiv 16 (1947) 72-76. 
[13] S. Ljunggren, Airborne sound insulation of thick walls, The Journal of the Acoustical Society of America 89 (1991) 2338-2345.

[14] S. Ljunggren, Forced vibrations of infinite plates, Journal of Sound and Vibration 121 (1988) 221-236.

[15] E. Carrera, An assessment of mixed and classical theories on global and local response of multilayered orthotropic plates, Composite Structures 50 (2000) 183-198.

[16] E. Carrera, Theories and finite elements for multilayered, anisotropic, composite plates and shells, Archives of Computational Methods in Engineering 9 (2002) 87-140.

[17] M. Levinson, An accurate, simple theory of the statics and dynamics of elastic plates, Mechanics Research Communications 7 (1980) 343-350.

[18] J. N. Reddy, A simple higher-order theory for laminated composite plates, Journal of Applied Mechanics 51 (1984) 745-752.

[19] M. Touratier, An efficient standard plate theory, International Journal of Engineering Science 29 (1991) 901-916.

[20] M. Karama, K. Afaq, S. Mistou, Mechanical behaviour of laminated composite beam by the new multi-layered laminated composite structures model with transverse shear stress continuity, International Journal of Solids and Structures 40 (2003) 1525-1546.

[21] X. Lu, D. Liu, Interlayer shear slip theory for cross-ply laminates with nonrigid interfaces, AIAA Journal 30 (1992) 1063-1073.

[22] C.-T. Sun, J. Whitney, Theories for the dynamic response of laminated plates, AIAA Journal 11 (1973) 178-183.

[23] R. Ford, P. Lord, A. Walker, Sound transmission through sandwich constructions, Journal of Sound and Vibration 5 (1967) 9-21.

[24] C. Smolenski, E. Krokosky, Dilational-mode sound transmission in sandwich panels, The Journal of the Acoustical Society of America 54 (1973) $1449-1457$.

[25] J. Moore, R. Lyon, Sound transmission loss characteristics of sandwich panel constructions, The Journal of the Acoustical Society of America 89 (1991) 777-791. 
[26] S. Narayanan, R. Shanbhag, Sound transmission through a damped sandwich panel, Journal of Sound and Vibration 80 (1982) 315-327.

[27] S. Ghinet, N. Atalla, Modeling thick composite laminate and sandwich structures with linear viscoelastic damping, Computers \& Structures 89 (2011) 1547-1561.

[28] S. Srinivas, A refined analysis of composite laminates, Journal of Sound and Vibration 30 (1973) 495-507.

[29] J.-L. Guyader, C. Lesueur, Acoustic transmission through orthotropic multilayered plates, Part I: Plate vibration modes, Journal of Sound and Vibration 58 (1978) 51-68.

[30] J.-L. Guyader, C. Lesueur, Acoustic transmission through orthotropic multilayered plates, part II: Transmission loss, Journal of Sound and Vibration 58 (1978) 69-86.

[31] C. Lee, D. Liu, Layer reduction technique for composite laminate analysis, Computers \& Structures 44 (1992) 1305-1315.

[32] R. L. Woodcock, Free vibration of advanced anisotropic multilayered composites with arbitrary boundary conditions, Journal of Sound and Vibration 312 (2008) 769-788.

[33] A. Loredo, A. Castel, A multilayer anisotropic plate model with warping functions for the study of vibrations reformulated from Woodcock's work, Journal of Sound and Vibration 332 (2013) 102-125.

[34] A. Loredo, A multilayered plate theory with transverse shear and normal warping functions, Composite Structures 156 (2016) 361-374.

[35] D. Ross, Damping of plate flexural vibrations by means of viscoelastic laminae, Structural Damping (1959) 49-97.

[36] E. M. Kerwin Jr, Damping of flexural waves by a constrained viscoelastic layer, The Journal of the Acoustical Society of America 31 (1959) 952962.

[37] E. E. Ungar, E. M. Kerwin Jr, Loss factors of viscoelastic systems in terms of energy concepts, The Journal of the Acoustical Society of America 34 (1962) 954-957. 
[38] J.-L. Guyader, C. Cacciolati, Viscoelastic properties of single layer plate material equivalent to multi-layer composites plate, in: Turkish Acoustical Society - 36th International Congress and Exhibition on Noise Control Engineering, Inter-Noise 2007 Istanbul, volume 3, 2007, pp. 15581567 .

[39] F. Marchetti, K. Ege, Q. Leclere, N. Roozen, On the structural dynamics of laminated composite plates and sandwich structures; a new perspective on damping identification, Journal of Sound and Vibration 474 (2020) 115256.

[40] Ö. Civalek, Harmonic differential quadrature-finite differences coupled approaches for geometrically nonlinear static and dynamic analysis of rectangular plates on elastic foundation, Journal of Sound and Vibration 294 (2006) 966-980.

[41] K. Bhaskar, T. Varadan, Plates: theories and applications, John Wiley \& Sons, 2014.

[42] J. Allard, N. Atalla, Propagation of Sound in Porous Media: Modelling Sound Absorbing Materials 2e, John Wiley \& Sons, 2009.

[43] G. Kirchhoff, Über Das Gleichgewicht Und Die Bewegung Einer Elastischen Scheibe, 1850.

[44] L. Cremer, M. Heckl, Structure-Borne Sound: Structural Vibrations and Sound Radiation at Audio Frequencies, Springer Science \& Business Media, 2013.

[45] E. Magrab, Vibrations of elastic structural members, Alphen aan den Rijn, Sijthoff and Noordhoff (1979).

[46] J.-M. Berthelot, F. F. Ling, Composite Materials: Mechanical Behavior and Structural Analysis, Springer, 1999.

[47] D. Folds, C. Loggins, Transmission and reflection of ultrasonic waves in layered media, The Journal of the Acoustical Society of America 62 (1977) 1102-1109.

[48] C. L. Dym, M. A. Lang, Transmission of sound through sandwich panels, The Journal of the Acoustical Society of America 56 (1974) 1523-1532.

[49] C. L. Dym, C. S. Ventres, M. A. Lang, Transmission of sound through sandwich panels: a reconsideration, The journal of the acoustical society of America 59 (1976) 364-367. 
[50] D. Rhazi, N. Atalla, A simple method to account for size effects in the transfer matrix method, The Journal of the Acoustical Society of America 127 (2010) EL30-EL36. 